\title{
Robust Moment-Based Energy-Maximising Optimal Control of Wave Energy Converters
}

\author{
Nicolás Faedo ${ }^{\mathrm{a}}$, Demián García-Violini ${ }^{\mathrm{a}}$, Giordano Scarciotti ${ }^{\mathrm{b}}$, Alessandro Astolfi ${ }^{\mathrm{b}, \mathrm{c}}$ and John V. Ringwood ${ }^{\mathrm{a}}$
}

\begin{abstract}
We introduce a moment-based framework to design robust energy-maximising optimal controllers for Wave Energy Converters (WECs). The technique explicitly allows model uncertainty in the computation of the optimal control input, by defining a suitable uncertainty polytope. The resulting robust optimisation is formulated as a minimax problem which has to be solved only at a small number of points of this uncertainty set. The objective function under the proposed strategy is shown to be of quadratic-type and the optimal solution is proven to be unique, providing a computationally efficient robust optimal control framework for WECs. The performance of the proposed controller is demonstrated by means of an application case, which considers a heaving point absorber WEC with imprecisely known model parameters.
\end{abstract}

\section{INTRODUCTION}

The energy-maximising control objective for WECs can be achieved by designing an optimal controller that maximises the time-averaged power extracted from ocean waves, while simultaneously ensuring that the intrinsic physical limitations of both the device and the Power-Take Off (PTO) system (actuator) are respected consistently. In summary, this control objective aims at successfully securing maximum power absorption with minimum risk of component damage. Several studies address this energy-maximising objective using a variety of techniques, as discussed, for example, in [1].

Though the number of control techniques proposed in the literature has increased considerably in recent years, only few studies (some cases reviewed in the next paragraph) address the robust design of energy-maximising controllers; the optimal control input is mostly computed based only on a nominal model, without considering possible dynamical deviations (uncertainties) [1]. By way of example, some parameters of the WEC hydrodynamic model, such as the viscous drag coefficient [2], can vary significantly due to the change in the relative motion of the device.

Recent studies that address the optimal control problem for WECs in a robust sense can be found in [3] and [4]. To briefly summarise, [3] takes into account possible deviations from the design model by proposing a nominal model predictive control strategy with an additional correction term in the exerted PTO force, as a function of the

\footnotetext{
anicolás Faedo, Demián García-Violini and John V. Ringwood are with the Centre for Ocean Energy Research, Maynooth University, Maynooth, Ireland nicolas.faedo@mu. ie

${ }^{\mathrm{b}}$ Giordano Scarciotti and Alessandro Astolfi are with the Department of Electrical and Electronic Engineering, Imperial College London, London SW7 2AZ, U.K

${ }^{\mathrm{c}}$ Alessandro Astolfi is with the Department of Civil Engineering and Computer Science Engineering, University of Rome “Tor Vergata”, 00133 Rome, Italy
}

(defined) uncertainty. The study performed in [4] utilises a spectral direct transcription technique and formulates the WEC control problem using a robust optimisation approach, based on key publications on this subject, such as [5].

Recently, an energy-maximising optimal control framework for WECs has been presented in [6], [7]. The mathematical formalism behind this strategy is based on the concept of moment (discussed herein in Section II), which maps the original optimal control problem into a concave Quadratic Program (QP), taking also into account motion (state and input) constraints. As a consequence, the constrained energy-maximising optimal control law can be obtained in a computationally efficient way using state-of-theart QP solvers, such as those described in [8]

In this study we propose an extension of the energymaximising moment-based framework proposed in [6] which explicitly considers model uncertainty in the computation of the optimal control law, while systematically respecting motion (state and input) constraints. In particular, we consider structured (parametric) uncertainty in the WEC design model, by defining a suitable convex polytope for the uncertainty set. To this end, we combine the concept of moment with the robust optimisation principles considered in [4], by proposing a Worst-Case Performance (WCP) approach (discussed in Section IV). The performance of the proposed controller is analysed by means of an application case considering a heaving point absorber WEC.

The remainder of this paper is organised as follows. Section II discusses key theoretical concepts behind the momentbased framework. Section III describes and formalises the energy-maximising problem for WECs, while Section IV details the proposed robust moment-based strategy. Finally, Section V discusses the application of the strategy for a spherical heaving point absorber WEC, whilst Section VI encompasses the main conclusions of this study.

\section{A. Notation and Preliminaries}

Standard notation is used throughout this study. $\mathbb{R}^{+}\left(\mathbb{R}^{-}\right)$ denotes the set of non-negative (non-positive) real numbers. $\mathbb{C}^{0}$ denotes the set of pure-imaginary complex numbers and $\mathbb{C}_{<0}$ denotes the set of complex numbers with negative real part. The symbol 0 stands for any zero element, dimensioned according to the context. The notation $\mathbb{N}_{q}$ indicates the set of all positive natural numbers up to $q$, i.e. $\mathbb{N}_{q}=\{1,2, \ldots, q\}$. The symbol $\mathbb{I}_{n}$ denotes the identity matrix of dimension $n \times n$, while the notation $\mathbf{1}_{n \times m}$ is used to denote a $n \times m$ Hadamard identity matrix (i.e. a $n \times m$ matrix with all its entries equal to 1 ). The spectrum of a matrix $A \in \mathbb{R}^{n \times n}$, i.e. 
the set of its eigenvalues, is denoted as $\lambda(A)$. The symbol $\bigoplus$ denotes the direct sum of $n$ matrices, i.e. $\bigoplus_{i=1}^{n} A_{i}=$ $\operatorname{diag}\left(A_{1}, A_{2}, \ldots, A_{n}\right)$. The vectorisation of $A \in \mathbb{C}^{n \times m}$ is written as $\operatorname{vec}\{A\}$. The Kronecker product between two matrices $M_{1} \in \mathbb{R}^{n \times m}$ and $M_{2} \in \mathbb{R}^{p \times q}$ is denoted by $M_{1} \otimes M_{2} \in \mathbb{R}^{n p \times m q}$. The Kronecker sum between two matrices $P_{1}$ and $P_{2}$, with $P_{1} \in \mathbb{R}^{n \times n}$ and $P_{2} \in \mathbb{R}^{k \times k}$, is denoted as $P_{1} \hat{\oplus} P_{2}$. The convex hull of a set of points $X=\left\{x_{1}, \ldots, x_{n}\right\} \subset \mathscr{X}$, where $\mathscr{X}$ is a finite dimensional Euclidean space, is denoted as $\operatorname{conv}\{X\}$. The convolution between two functions $f$ and $g$ over the finite range $[0, t]$, i.e. $\int_{0}^{t} f(\tau) g(t-\tau) d \tau$, is denoted as $f * g$. The symbol $e_{i j}^{q} \in \mathbb{R}^{q \times q}$ denote a matrix with 1 in the $i j$ entry and 0 elsewhere. Likewise, $e_{i}^{q} \in \mathbb{R}^{q}$ denotes a vector with 1 in the $i$ entry and 0 elsewhere. Finally, the symbol $\varepsilon_{n} \in \mathbb{R}^{n \times 1}$ denotes a vector with odd entries equal to 1 and even entries equal to 0 .

\section{MOMEnT-BASED THEORY}

This section briefly recalls key concepts behind the moment-based framework, as developed in [9]. Consider a finite-dimensional, single-input, single-output, continuoustime system described, for $t \in \mathbb{R}^{+}$, by the state-space model

$$
\dot{x}(t)=A x(t)+B u(t), \quad y(t)=C x(t),
$$

where $x(t) \in \mathbb{R}^{n}, u(t) \in \mathbb{R}, y(t) \in \mathbb{R}, A \in \mathbb{R}^{n \times n}, B \in \mathbb{R}^{n}$ and $C \in \mathbb{R}^{1 \times n}$, and assume that (1) is minimal.

Lemma 1. [9] Consider system (1) and the autonomous signal generator

$$
\dot{\xi}(t)=S \xi(t), \quad u(t)=L \xi(t),
$$

with $\xi(t) \in \mathbb{R}^{\nu}, S \in \mathbb{R}^{\nu \times \nu}, L \in \mathbb{R}^{1 \times \nu}$ and $\xi(0) \in \mathbb{R}^{\nu}$. Assume that the triple $(L, S, \xi(0))$ is minimal, $\lambda(A) \subset \mathbb{C}_{<0}$, $\lambda(S) \subset \mathbb{C}^{0}$ and the eigenvalues of $S$ are simple. Then there is a unique matrix $\Pi \in \mathbb{R}^{n \times \nu}$ which solves the Sylvester equation

$$
A \Pi+B L=\Pi S,
$$

and the steady-state response of the output of the interconnected system (1)-(2) is $y_{s s}(t)=C \Pi \xi(t)$.

Remark 1. The minimality of the triple $(L, S, \xi(0))$ implies the observability of $(L, S)$ and the excitability ${ }^{1}$ of $(S, \xi(0))$.

Definition 1. The matrix $C \Pi$, with $\Pi$ solution of the Sylvester equation (3), is the moment of system (1) at the signal generator (2).

Remark 2. From now on, we refer to the matrix $\underline{Y}=C \Pi$ as the moment-domain equivalent of $y(t)$.

\section{ENERGY-MAXIMISING CONTROL OF WECS: PRELIMINARIES}

As discussed in [1], [6], the energy-maximising control criterion for WECs can be generally posed in terms of an

\footnotetext{
${ }^{1}$ We refer the reader to [9] for the definition of excitability.
}

objective function $\mathcal{J}$, which takes into account the useful energy converted by the PTO in the time interval $[0, T]$, i.e.

$$
\mathcal{J}=\frac{1}{T} \int_{0}^{T} u(\tau) \dot{x}(\tau) d \tau
$$

where $u$ and $\dot{x}$ denote the velocity of the device and the PTO control force, respectively. The calculation of the control law optimising (4) is non-trivial, mainly due to the irregularity of the poly-chromatic input, i.e. the wave excitation force (see Section III-A), to the system (see, for example, [1]).

\section{A. WEC dynamics}

The linearised equation of motion for a 1 degree-offreedom device can be expressed as follows:

$$
m \ddot{x}(t)=\mathcal{F}_{r}(t)+\mathcal{F}_{h}(t)+\mathcal{F}_{v}(t)+\mathcal{F}_{e}(t)-u(t),
$$

where $m$ is the mass of the buoy, $x$ the device excursion, $\mathcal{F}_{e}$ the wave excitation force, $\mathcal{F}_{h}$ the hydrostatic restoring force, $\mathcal{F}_{v}$ the viscous drag force, $\mathcal{F}_{r}$ the radiation force, and $u$ the control (PTO) force. The linearised hydrostatic force can be written as $\mathcal{F}_{h}(t)=-s_{h} x(t)$, where $s_{h}>0$ denotes the hydrostatic stiffness. The radiation force $\mathcal{F}_{r}$ is modelled based on linear potential theory and, using the well-known Cummins' equation [10], can be written as

$$
\mathcal{F}_{r}(t)=-\mu_{\infty} \ddot{x}(t)-\int_{0}^{+\infty} k(\tau) \dot{x}(t-\tau) d \tau,
$$

where $\mu_{\infty}=\lim _{\omega \rightarrow+\infty} \tilde{A}(\omega), \mu_{\infty}>0$, with $\tilde{A}(\omega)$ the radiation added mass ${ }^{2}$, represents the added-mass at infinite frequency, and $k$ is the (causal) radiation impulse response. The linearised drag force can be written as $\mathcal{F}_{v}(t)=b_{v} \dot{x}(t)$, with $b_{v}=\frac{1}{2} \rho D C_{d} \gamma$, where $\rho$ is the water density, $D$ is the characteristic area of the device, $C_{d}>0$ is the viscous drag force coefficient and $\gamma$ represents the linearisation coefficient. Finally, the linearised equation of motion is given by

$\left(m+\mu_{\infty}\right) \ddot{x}(t)+k(t) * \dot{x}(t)+b_{v} \dot{x}(t)+s_{h} x(t)=\mathcal{F}_{e}(t)-u(t)$,

The internal stability of equation (7), for the WEC case, has been analysed and guaranteed for any physically meaningful value of the parameters and the convolution kernel $k$ involved [11].

\section{B. Optimal control formulation and motion constraints}

We consider constraints on both the displacement of the WEC $x$ and the exerted control (PTO) force $u$, which can be compactly written as

$$
\left\{\begin{array}{l}
|x(t)| \leq X_{\max }, \quad \forall t \in \mathbb{R}^{+},\left\{X_{\max }, U_{\max }\right\} \subset \mathbb{R}^{+} . \\
|u(t)| \leq U_{\max },
\end{array}\right.
$$

Given the objective function defined in (4), the governing dynamics of the WEC in (7) and the set of state and input

\footnotetext{
${ }^{2}$ See [11] for the definition of $\tilde{A}(\omega)$.
} 
constraints defined in (8), the constrained energy-maximising optimal control problem can be stated as

$$
\begin{gathered}
u^{\mathrm{opt}}(t)=\arg \max _{u(t) \in \mathbb{R}} \mathcal{J} \\
\text { subject to: }
\end{gathered}
$$$$
\left\{\begin{array}{l}
\text { WEC dynamics (7), } \\
\text { state and input constraints (8). }
\end{array}\right.
$$

\section{Moment-Based WEC Control: Robust FORMULATION}

In the robust moment-based framework proposed in this paper we consider parametric uncertainties in (7). In particular, we assume that the parameters $m, b_{v}$ and $s_{h}$ are imprecisely known quantities, written as

$$
m=m^{0}+m^{\delta}, \quad b_{v}=b_{v}^{0}+b_{v}^{\delta}, \quad s_{h}=s_{h}^{0}+s_{h}^{\delta},
$$

where $m^{0}, b_{v}^{0}$, and $s_{h}^{0}$ denote the nominal parameter values and the elements of the vector $\delta=\left[m^{\delta}, b_{v}^{\delta}, s_{h}^{\delta}\right]^{\top} \in \mathcal{P}$ are variations around the nominal values. It is assumed that $\mathcal{P} \subset$ $\mathbb{R}^{3}$ is a convex $\mathcal{V}$-polytope (the reader is referred to [12] for further detail) given as the convex hull of a finite set of $N$ points (vertices) in space $V_{\delta}=\left\{\delta_{1}^{V}, \ldots, \delta_{N}^{V}\right\}$, i.e. $\mathcal{P}=\operatorname{conv}\left\{V_{\delta}\right\}$.

Considering the equation of motion (7), the imprecisely known parameters described in (10) and defining the function

$$
\mathscr{U}(t)=\mathcal{F}_{e}(t)-u(t)-k(t) * \dot{x}(t),
$$

we write the uncertain system dynamics in standard $\Sigma-\Delta$ configuration [12] with

$$
\begin{gathered}
\mathscr{D}(t)=\Delta \mathscr{Z}(t), \quad \Delta=\left[\begin{array}{ccc}
m^{\delta} & 0 & 0 \\
0 & b_{v}^{\delta} & 0 \\
0 & 0 & s_{h}^{\delta}
\end{array}\right], \\
\Sigma:\left\{\begin{array}{l}
\dot{\varphi}(t)=A^{0} \varphi(t)+B^{0} \mathscr{U}(t)+D^{0} \mathscr{D}(t), \\
\mathscr{Z}(t)=A_{\mathscr{Z}} \varphi(t)+B_{\mathscr{Z}} \mathscr{U}(t)+D_{\mathscr{Z}} \mathscr{D}(t), \\
y_{\varphi}(t)=C \varphi(t),
\end{array}\right.
\end{gathered}
$$

where $\varphi(t)=[x(t), \dot{x}(t)]^{\top} \in \mathbb{R}^{2}$ and $y_{\varphi}(t)=\dot{x}(t) \in \mathbb{R}$ is the output of the system (the device velocity). The matrices of the dynamical system $\Sigma$ in (13) are defined as

$$
\begin{aligned}
A^{0}= & {\left[\begin{array}{cc}
0 & 1 \\
-\frac{s_{h}^{0}}{m^{0}+\mu_{\infty}} & -\frac{b_{v}^{0}}{m^{0}+\mu_{\infty}}
\end{array}\right], B^{0}=\left[\begin{array}{c}
0 \\
\frac{1}{m^{0}+\mu_{\infty}}
\end{array}\right], } \\
A_{\mathscr{Z}}= & {\left[\begin{array}{cc}
-\frac{s_{h}^{0}}{m^{0}+\mu_{\infty}} & -\frac{b_{v}^{0}}{m^{0}+\mu_{\infty}} \\
0 & 1 \\
1 & 0
\end{array}\right], B_{\mathscr{Z}}=\left[\begin{array}{c}
\frac{1}{m^{0}+\mu_{\infty}} \\
0 \\
0
\end{array}\right], } \\
D^{0}= & {\left[\begin{array}{ccc}
0 & 0 \\
-\frac{1}{m^{0}+\mu_{\infty}} & -\frac{1}{m^{0}+\mu_{\infty}} & -\frac{1}{m^{0}+\mu_{\infty}}
\end{array}\right], } \\
D_{\mathscr{Z}} & =\left[\begin{array}{ccc}
-\frac{1}{m^{0}+\mu_{\infty}} & -\frac{1}{m^{0}+\mu_{\infty}} & -\frac{1}{m^{0}+\mu_{\infty}} \\
0 & 0 & 0 \\
0 & 0 & 0
\end{array}\right], \\
C & =\left[\begin{array}{ccc}
0 & 1
\end{array}\right] .
\end{aligned}
$$

Remark 3. The matrix $\Delta$ can be explicitly written as a function of $\delta \in \mathcal{P}$, i.e. $\Delta=g(\delta)$, with $g(\delta)=\sum_{i=1}^{3} e_{i i}^{3} \delta e_{i}^{3}$.
Following the theoretical basis of the moment-based framework, both the wave excitation force $\mathcal{F}_{e}$ and the control input $u$ are expressed as output of a signal generator as

$$
\dot{\xi}(t)=S \xi(t), \quad \mathcal{F}_{e}(t)=L_{e} \xi(t), \quad u(t)=L_{u} \xi(t),
$$

where the dimension of $S, L_{e}$ and $L_{u}$ are as in (2), $\xi(t) \in \mathbb{R}^{\nu}$ and the pairs $\left(L_{e}, S\right)$ and $\left(L_{u}, S\right)$ are observable. Given the characteristics of $\lambda(S)$ (see Lemma 1), we consider the finite set $\mathscr{F}=\left\{\omega_{p}\right\}_{p=1}^{f} \subset \mathbb{R}$ and write the matrix $S$ in a real block-diagonal form as

$$
S=\bigoplus_{p=1}^{f}\left[\begin{array}{cc}
0 & \omega_{p} \\
-\omega_{p} & 0
\end{array}\right]
$$

where $\nu=2 f, f>0$ integer. Without any loss of generality, the initial condition of the signal generator is $\xi_{e}(0)=\varepsilon_{\nu}$.

With this selection of matrices, the moment of the uncertain system (13), driven by the signal generator (15), can be computed by solving the Sylvester equation (see Lemma 1)

$$
\left(A^{0}+A^{\Delta}\right) \Pi_{\varphi}^{\Delta}+\left(B^{0}+B^{\Delta}\right)\left(L_{e}-L_{u}-\underline{\mathcal{Z}}\right)=\Pi_{\varphi}^{\Delta} S,
$$

where $\Pi_{\varphi}^{\Delta} \in \mathbb{R}^{2 \times \nu}$ and $\underline{\mathcal{Z}}$ is the moment-domain equivalent of the radiation force convolution term (see [6]). The matrices $A^{\Delta}$ and $B^{\Delta}$ can be computed immediately, after algebraic manipulation of (13), and are defined as

$$
\begin{aligned}
& A^{\Delta}=D^{0} \Delta\left(\mathbb{I}_{3}-D_{\mathscr{Z}} \Delta\right)^{-1} A_{\mathscr{Z}}, \\
& B^{\Delta}=D^{0} \Delta\left(\mathbb{I}_{3}-D_{\mathscr{Z}} \Delta\right)^{-1} B_{\mathscr{Z}} .
\end{aligned}
$$

Note that the moment-domain equivalent of the velocity can be expressed in terms of the solution of (17), straightforwardly, as $\underline{\mathcal{V}}^{\Delta}=C \Pi_{\varphi}^{\Delta}$.

We now state the following proposition, that establishes the uniqueness of the solution of the Sylvester equation (17) associated with the uncertain system $\Sigma$.

Proposition 1. Suppose $\Delta=g(\delta)$, with $\delta \in \mathcal{P}$, is such that system $\Sigma$ in (13) is asymptotically stable. Then the momentdomain equivalent of the output $y_{\varphi}$ of system (13) is uniquely determined as

$$
\underline{\mathcal{V}}^{\Delta}=\left(L_{e}-L_{u}\right) \Psi_{\varphi}^{\Delta \top},
$$

where

$$
\begin{aligned}
& \Psi_{\varphi}^{\Delta}=\left(\mathbb{I}_{\nu} \otimes C\right) \Phi_{\varphi}^{\Delta^{-1}}\left(\mathbb{I}_{\nu} \otimes\left(B^{0}+B^{\Delta}\right)\right), \\
& \Phi_{\varphi}^{\Delta}=\left(S \hat{\oplus}\left(A^{0}+A^{\Delta}\right)\right)+\mathscr{R}^{\top} \otimes-\left(B_{0}+B^{\Delta}\right) C,
\end{aligned}
$$

with $\Phi_{\varphi}^{\Delta} \in \mathbb{R}^{2 \nu \times 2 \nu}, \Psi_{\varphi}^{\Delta} \in \mathbb{R}^{\nu \times \nu}$ and where $\mathscr{R} \in \mathbb{R}^{\nu \times \nu}$ is a block-diagonal matrix defined as

$$
\begin{aligned}
\mathscr{R} & =\bigoplus_{p=1}^{f}\left[\begin{array}{cc}
r_{\omega_{p}} & m_{\omega_{p}} \\
-m_{\omega_{p}} & r_{\omega_{p}}
\end{array}\right], \\
\text { with } \quad r_{\omega_{p}} & =\tilde{B}\left(\omega_{p}\right), \quad m_{\omega_{p}}=\omega_{p}\left[\tilde{A}\left(\omega_{p}\right)-\mu_{\infty}\right],
\end{aligned}
$$

where $\tilde{A}(\omega)$ is the radiation added-mass and $\tilde{B}(\omega)$ is the radiation damping ${ }^{3}$ of the device at each specific frequency associated with the eigenvalues of the matrix $S$.

\footnotetext{
${ }^{3}$ See [11] for the definition of $\tilde{B}(\omega)$.
} 
The result of Proposition 1 provides a method both to guarantee the uniqueness and to perform the computation of the moment-domain equivalent of the uncertain system $\Sigma$ defined in (13). Using these theoretical results, and following a similar analysis to that of [6] for the nominal WEC model case, we solve the moment-based formulation of the energymaximising optimal control problem (9) when considering the uncertain system $\Sigma$.

Proposition 2. Consider the (motion unconstrained) energymaximising optimal control problem (9). Let $\omega_{p}=p \omega_{0}$ in (16) for all $p \in \mathbb{N}_{f}$. Then the optimal control law $u^{\text {opt }}(t)=$ $L_{u}^{\text {opt }} \xi(t)$ that maximises $\mathcal{J}$ over the time period $[0, T]$, with $T=2 \pi / \omega_{0}$, can be computed in the moment-domain as the solution of the $Q P$ problem

$$
L_{u}^{o p t}=\arg \max _{L_{u} \in \mathbb{R}^{1 \times \nu}}-\frac{1}{2} L_{u} \Psi_{\varphi}^{\Delta \top} L_{u}^{\top}+\frac{1}{2} L_{e} \Psi_{\varphi}^{\Delta \boldsymbol{\top}} L_{u}^{\top} .
$$

Proposition 3. The QP optimisation problem defined in (23) has a unique global solution if and only if

$$
b_{v}^{\Delta}+b_{v}^{0}+r_{\omega_{p}}>0, \text { for all } p \in \mathbb{N}_{f}
$$

Proposition 3 states that the existence of a unique global maximiser for the optimal control input under the QP problem (23) is completely determined by three particular quantities: the radiation damping of the device at the frequencies associated with the eigenvalues of the matrix $S$, and the nominal value and the uncertainty of the viscous force coefficients, i.e. $b_{v}^{0}$ and $b_{v}^{\delta}$, respectively.

\section{A. Robust optimisation problem}

In this section, following the approach proposed in [4], we re-formulate the moment-domain optimisation problem (23) based on the underlying principles of robust optimisation theory developed in key studies, such as [5]. The underpinning concept behind this approach originates in the field of decision theory and is known as the Worst-Case Performance (WCP) method. For our moment-domain QP problem in equation (23), the (well-posed) robust QP formulation can be defined as follows.

Problem 1 (Robust formulation). Suppose condition (24) holds for every $\delta \in \mathcal{P}$ and every $p \in \mathbb{N}_{f}$. Then the robust formulation of the (motion unconstrained) QP problem (23) can be written as

$L_{u}^{R C}=\arg \max _{L_{u} \in \mathbb{R}^{1 \times \nu}} \min _{\delta \in \mathcal{P}}-\frac{1}{2} L_{u} \Psi_{\varphi}^{\Delta \top} L_{u}^{\top}+\frac{1}{2} L_{e} \Psi_{\varphi}^{\Delta \boldsymbol{\top}} L_{u}^{\top}$,

where $u^{R C}(t)=L_{u}^{R C} \xi(t)$ denotes the worst-case performance energy-maximising optimal control law.

Problem 1 computes the worst-case scenario for the energy-maximising problem (23) taking into account every possible uncertainty $\delta$ lying inside the polytope $\mathcal{P}$.

Remark 4. Standard nonlinear programming routines can be used to solve (25). Moreover, given the concavity of the QP problem and the convexity of the polytope $\mathcal{P}$, the solution of (25) always lies precisely at one of the vertices of $\mathcal{P}$ [8], [13], i.e. it is sufficient to solve equation (25) only for the $N$ elements of the finite set $V_{\delta}$.

\section{B. Handling of motion constraints}

The set of motion constraints (8) can be mapped to their respective moment-domain equivalents following the theory developed in [6], as ${ }^{4}$

$$
\left\{\begin{array}{l}
|x(t)| \leq X_{\max }, \\
|u(t)| \leq U_{\max }
\end{array}, \mapsto\left\{\begin{array}{l}
\left|\underline{\mathcal{V}}^{\Delta} S^{-1} e^{S t} \varepsilon_{\nu}\right| \leq X_{\max } \\
\left|L_{u} e^{S t} \varepsilon_{\nu}\right| \leq U_{\max }
\end{array}\right.\right.
$$

to then be enforced only at a set of specified time instants $\mathcal{T}=\left\{t_{i}\right\}_{i=1}^{N_{c}} \subset \mathbb{R}^{+}$. Given that it is sufficient to solve the robust formulation (25) only at the finite set of vertices of the convex polytope $\mathcal{P}$ (see Remark 4), a sensible approach to incorporate state constraints into Problem 1 is to guarantee that such constraints are satisfied at every point of the set $V_{\delta}$. This effectively ensures that (8) is consistently fulfilled for every $\delta \in \mathcal{P}$ [8], [13].

By defining the matrices $\Lambda \in \mathbb{R}^{\nu \times N_{c}}$ and $\Omega \in \mathbb{R}^{\nu \times 2 N_{c}}$ as

$$
\Lambda=\left[\begin{array}{lll}
\left(e^{S t_{1}} \varepsilon_{\nu}\right) & \cdots & \left(e^{S t_{N_{c}}} \varepsilon_{\nu}\right)
\end{array}\right], \Omega=\left[\begin{array}{ll}
\Lambda & -\Lambda
\end{array}\right]
$$

and substituting $\underline{\mathcal{V}}^{\Delta}$ from (19) in the (mapped) constraints of equation (26), the motion constrained robust energymaxisimising optimal control problem can be written in the moment-domain as an inequality-constrained minimax QP problem.

Problem 2 (Constrained robust formulation). Suppose condition (24) holds for every $\delta \in \mathcal{P}$ and every $p \in \mathbb{N}_{f}$. Then the motion constrained robust QP formulation is given by

$$
\begin{aligned}
& L_{u}^{R C}=\arg \max _{L_{u} \in \mathbb{R}^{1 \times \nu}} \min _{\delta \in V_{\delta} \subset \mathcal{P}}-\frac{1}{2} L_{u} \Psi_{\varphi}^{\Delta \top} L_{u}^{\top}+\frac{1}{2} L_{e} \Psi_{\varphi}^{\Delta \top} L_{u}^{\top}, \\
& \quad \text { subject to: } \\
& \quad L_{u} \mathcal{A}_{x}^{R C} \leq \mathcal{B}_{x}^{R C}, \quad L_{u} \mathcal{A}_{u}^{R C} \leq \mathcal{B}_{u}^{R C},
\end{aligned}
$$

where

$$
\begin{array}{ll}
\mathcal{A}_{x}^{R C}=\left[\mathcal{A}_{x}^{\delta_{1}}, \ldots, \mathcal{A}_{x}^{\delta_{N}}\right], & \mathcal{B}_{x}^{R C}=\left[\mathcal{B}_{x}^{\delta_{1}}, \ldots, \mathcal{B}_{x}^{\delta_{N}}\right], \\
\mathcal{A}_{u}^{R C}=\Omega, & \mathcal{B}_{u}^{R C}=U_{\max } \mathbf{1}_{1 \times 2 N_{c}},
\end{array}
$$

and the matrices $\mathcal{A}_{x}^{\delta_{i}}, \mathcal{B}_{x}^{\delta_{i}}$ are defined as

$$
\mathcal{A}_{x}^{\delta_{i}}=-\Psi_{\varphi}^{\Delta_{i} \top} S^{-1} \Omega, \mathcal{B}_{x}^{\delta_{i}}=X_{\max } \mathbf{1}_{1 \times 2 N_{c}}+L_{e} \mathcal{A}_{x}^{\delta_{i}},
$$

where $\Psi_{\varphi}^{\Delta_{i}}$ is computed as $\Psi_{\varphi}^{\Delta}$ in (20) with $\Delta=g\left(\delta_{i}\right)$, $\delta_{i} \in V_{\delta}$, for all $i \in \mathbb{N}_{N}$ (we refer the reader to Remark 3 for the definition of the mapping $g$ ).

Remark 5. The computation of the matrices $\mathcal{A}_{u}^{\mathrm{RC}}$ and $\mathcal{B}_{u}^{\mathrm{RC}}$ can be performed independently from the polytope $\mathcal{P}$, i.e. the definition of the PTO force constraint does not depend on the uncertainty set. Though this is not the case with the matrices $\mathcal{A}_{x}^{\mathrm{RC}}, \mathcal{B}_{x}^{\mathrm{RC}}$, which explicitly depend on the nature of the polytope $\mathcal{P}$ (i.e. the set of vertices $V_{\delta}$ ), we note that it is sufficient to compute both matrices only once, which can be readily done offline after the definition of the set $\mathcal{P}$.

\footnotetext{
${ }^{4}$ Note that the moment-domain equivalent of the position $x$ can be expressed as $\underline{\mathcal{V}}^{\Delta} S^{-1}[9]$.
} 


\section{Applichtion to a Heaving Point Absorber WEC}

We consider a spherical heaving point absorber WEC with a diameter of $5[\mathrm{~m}]$, subject both to regular (monochromatic) and irregular (polychromatic) waves. The nominal values for the model parameters involved in $\Sigma$, i.e. equation (13), are set to $m^{0}=30.34 \times 10^{3}[\mathrm{~kg}], b_{v}^{0}=29.45 \times 10^{3}[\mathrm{~kg} / \mathrm{s}]$ and $s_{h}^{0}=196.87 \times 10^{3}\left[\mathrm{~kg} / \mathrm{s}^{2}\right]$.

It is now useful to write the nominal parameter $b_{v}^{0}$ as $b_{v}^{0}=$ $\frac{1}{2} \rho A_{d} C_{d}^{0} \gamma^{0}$, where $C_{d}^{0}$ and $\gamma^{0}$ stand for the nominal viscous drag and nominal linearisation coefficients, respectively. To determine the polytope $\mathcal{P}$, we assume that both $C_{d}$ and $\gamma$ are uncertain, specifically within $\pm 10 \%$ of their respective nominal values. In this study we make explicit use of our robust moment-based strategy to overcome the important drawback of choosing a fixed value for $C_{d}$ and $\gamma$ (see, for example, [2], [14]), by allowing these quantities to vary over a suitably defined convex polytope $\mathcal{P}$. For this example case we assume that both $C_{d}$ and $\gamma$ vary $\pm 10 \%$ around each of their respective nominal values. Following the definition of $\Delta$ in (13), these uncertainty levels can be covered in this robust approach by assuming that the viscous force coefficient $b_{v}$ can vary within a range of $\pm 25 \%$ of its nominal value $b_{v}^{0}$. Finally, we consider that the hydrodynamic stiffness $s_{h}$ can vary within $\pm 15 \%$ of its nominal value $s_{h}^{0}$, and that there is no uncertainty in the mass of the device ${ }^{5}$.

To be precise, we define the polytope $\mathcal{P}$ as the convex hull of the set $V_{\delta}=\left\{\delta_{1}^{V}, \delta_{2}^{V}, \delta_{3}^{V}, \delta_{4}^{V}\right\}$ with vertices

$$
\begin{aligned}
& \delta_{1}^{V}=\left[0,-0.25 b_{v}^{0}, 0.15 s_{h}^{0}\right]^{\top}, \\
& \delta_{2}^{V}=\left[0,0.25 b_{v}^{0}, 0.15 s_{h}^{0}\right]^{\top}, \\
& \delta_{3}^{V}=\left[0,0.25 b_{v}^{0},-0.15 s_{h}^{0}\right]^{\top}, \\
& \delta_{4}^{V}=\left[0,-0.25 b_{v}^{0},-0.15 s_{h}^{0}\right]^{\top} .
\end{aligned}
$$

This polytope is depicted in Figure 1, where each vertex $\delta_{i}^{V}$ is specified by a white circle. In addition to the set $V_{\delta}$, Figure 1 indicates two important elements contained in the polytope $\mathcal{P}: \delta^{0}$ (indicated with a solid-black dot in Figure 1) and $\delta^{\star}$ (indicated with a green star in Figure 1). The former defines the nominal system $\Sigma^{0}$, i.e. system $\Sigma$ in (13) when $\Delta=$ $g\left(\delta^{0}\right)=0$, while the latter defines the position of the actual system $\Sigma^{\star}$ inside the polytope, i.e. system $\Sigma$ with $\Delta=g\left(\delta^{\star}\right)$. For the following case, $\delta^{\star}=\left[0,0.1 b_{v}^{0},-0.2 s_{h}^{0}\right] \in \mathcal{P}$.

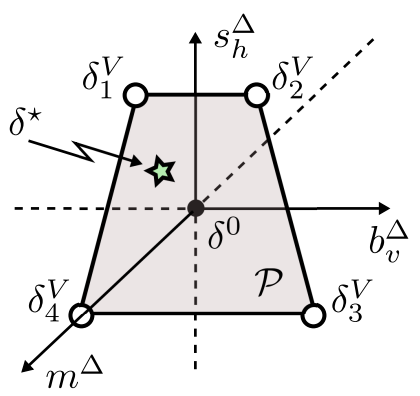

Fig. 1. Convex polytope $\mathcal{P} \subset \mathbb{R}^{3}$ considered for the case study.

\footnotetext{
${ }^{5}$ This assumption does not pose any loss of generality. It is only considered to simplify the definition of the convex polytope $\mathcal{P}$ and its corresponding visualisation in Figure 1.
}

We now define three scenarios, which are consistently considered in the remainder of this section. Note that we also provide the color and line codes used in the upcoming figures for each particular scenario.

...... Ideal performance: the optimal control input is computed using the nominal approach, i.e. using the nominal model $\Sigma^{0}$ and without considering any possible uncertainty, and applied to the same nominal system.

- - $\boldsymbol{-}$ - Nominal performance: the optimal control input is computed using the nominal approach, i.e. using the nominal model $\Sigma^{0}$ and without considering any possible uncertainty, and applied to the actual system $\Sigma^{\star}$.

Robust performance: the optimal control input is computed using the robust approach proposed in this paper, i.e. the control law explicitly uses the knowledge of the uncertainty polytope $\mathcal{P}$, and applied to the actual system $\Sigma^{\star}$.

We first assess the performance of the robust momentbased controller in regular waves without motion constraints. Figure 2 shows the value of the objective function $\mathcal{J}$ (absorbed power) for several wave periods $T$ [s], with $T \in$ $[3,12]$, and wave height $H \in[1,3]$ [m]. It can be readily appreciated that the robust strategy outperforms the nominal case when applied to the actual model $\Sigma^{\star}$, being able to control the WEC device reasonably close to its maximum achievable performance for the complete set of the analysed wave periods and different wave heights.

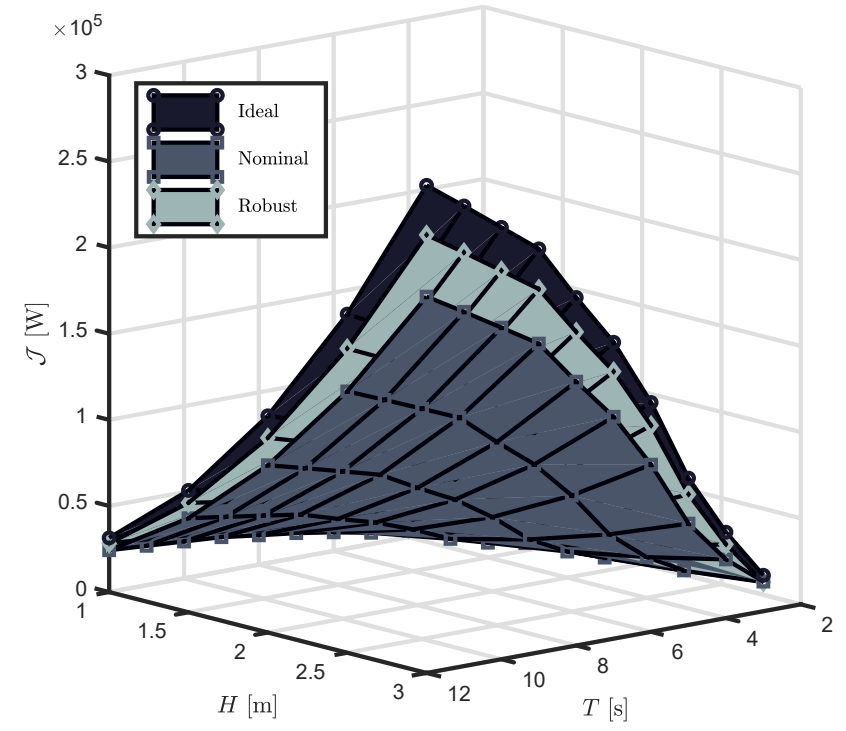

Fig. 2. Value of the objective function $\mathcal{J}$ for the ideal, nominal and robust performance cases, with varying wave period $T$ and wave height $H$.

In what follows we analyse the performance of the robust moment-based controller under polychromatic excitation and with active motion (state and input) constraints. The displacement and control input constraints are set to $X_{\max }=1$ [m] and $U_{\max }=1 \times 10^{5}[\mathrm{~N}]$, respectively. To generate the irregular waves case, we consider a JONSWAP spectrum [15] with a significant wave height of 3 [m], peak wave period of 8 [s] and peak enhancement factor of 3.3. Figure 3 shows 
both the device displacement (top) and velocity (centre) in the nominal and robust performance cases. One of the key aspects to highlight in the device displacement case is the fact that in the nominal performance case the displacement constraint is violated at several time instants. Though this constraint violation is not significant (in magnitude) for this example case, it happens consistently in time and can potentially damage the device components. Unlike the nominal performance case, when the robust optimal control input is applied to the actual system, the displacement limitation of \pm 1 [m] is consistently respected over the complete simulation time, as a consequence of the definition of the constraints in the optimisation problem (25). In the case of the device velocity, both scenarios perform similarly, preserving the well-known in-phase ${ }^{6}$ phenomenon with the excitation force (depicted in dotted-blue line), coinciding with the results of optimal energy absorption for unconstrained motion in regular waves (see [11]). Lastly, Figure 3 (bottom) depicts the optimal control laws computed for both the nominal and the robust performance cases. Note that both control laws always respect the PTO force limitation, as a consequence of the fact that such a constraint is formulated independently from the definition of the uncertainty (see Remarks 6 and 7).
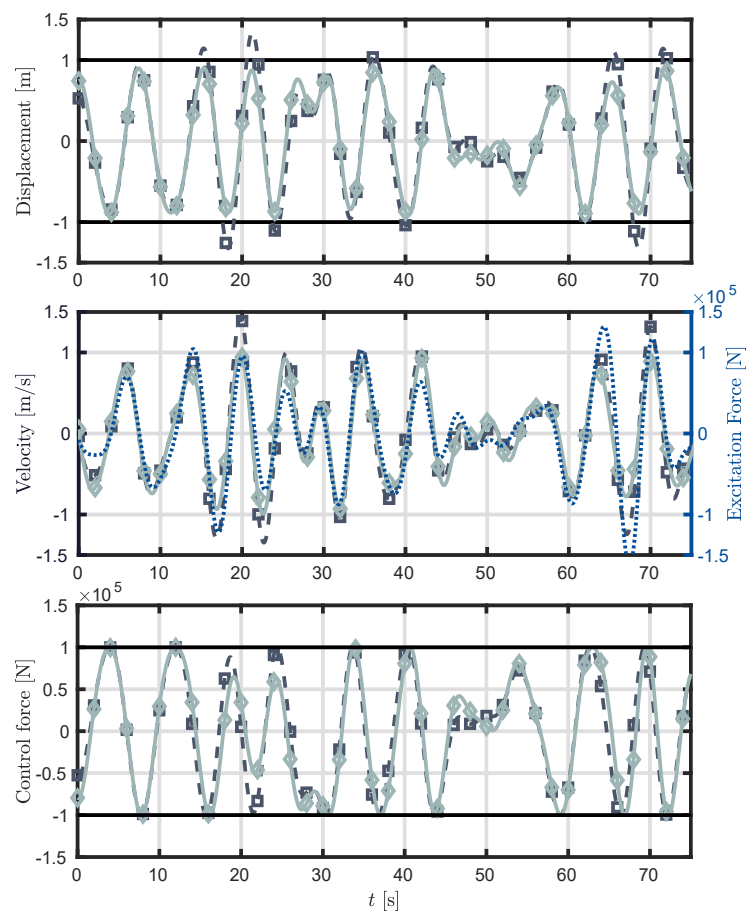

Fig. 3. Device displacement (top), velocity (centre) and optimal control force (bottom) for the ideal, nominal and robust performance cases, under state and input constraints. In the velocity figure, the wave excitation force $\mathcal{F}_{e}$ is depicted, using a dotted-blue line.

\section{CONCLUSIONS}

This study proposes a moment-based energy-maximising technique which allows the user to consider model uncer-

\footnotetext{
${ }^{6}$ The concept of phase is not defined for a signal with multiple frequency components. Herein, we use the expression "in-phase" to denote that the peaks (maxima and minima) of both signals are aligned in time.
}

tainty in the computation of the energy-maximising optimal control input. This robust approach effectively incorporates parametric uncertainty in the WEC moment-based energymaximising optimisation problem, by means of a suitable definition of an uncertainty (convex) polytope, and exploiting the underpinning concept of the Worst-Case Performance method. The proposed optimisation is formulated as a minimax problem which has to be solved only at the vertices of the polytope. This is due to the concavity of the objective function obtained once the state variables and both the external and control inputs are mapped to the moment-domain. As a result, this strategy provides a computationally efficient robust optimal control law which is able to consistently satisfy state and input constraints under the presence of parametric uncertainty.

\section{ACKNOWLEDGMENT}

This material is based upon works supported by Science Foundation Ireland under Grant no. 13/IA/1886 and the Royal Society International Exchange Cost Share programme (IEC $\backslash \mathrm{R} 1 \backslash 180018$ ). This work has been partially supported by the European Union's Horizon 2020 Research and Innovation Programme under grant agreement No 739551 (KIOS $\mathrm{CoE})$.

\section{REFERENCES}

[1] N. Faedo, S. Olaya, and J. V. Ringwood, "Optimal control, MPC and MPC-like algorithms for wave energy systems: An overview," IFAC Journal of Systems and Control, vol. 1, pp. 37-56, 2017.

[2] G. Giorgi and J. V. Ringwood, "Consistency of viscous drag identification tests for wave energy applications," in Proceedings of the 12th European Wave and Tidal Energy Conference (EWTEC). Cork, 2017.

[3] M. Jama, A. Wahyudie, and H. Noura, "Robust predictive control for heaving wave energy converters," Control Engineering Practice, vol. 77, pp. 138-149, 2018.

[4] D. Garcia-Violini and J. V. Ringwood, "Energy maximising robust control for spectral and pseudospectral methods with application to wave energy systems," International Journal of Control, 2019.

[5] A. Ben-Tal and A. Nemirovski, "Robust convex optimization," Mathematics of operations research, vol. 23, no. 4, pp. 769-805, 1998.

[6] N. Faedo, G. Scarciotti, A. Astolfi, and J. V. Ringwood, "Energymaximising control of wave energy converters using a moment-domain representation," Control Engineering Practice, vol. 81, pp. 85 - 96, 2018.

[7] _ - "Moment-based constrained optimal control of an array of wave energy converters," in 2019 American Control Conference (ACC), Philadelphia, 2019, pp. 4797-4802.

[8] S. Boyd and L. Vandenberghe, Convex optimization. Cambridge university press, 2004.

[9] G. Scarciotti and A. Astolfi, "Nonlinear model reduction by moment matching," Foundations and Trends in Systems and Control, vol. 4, no. 3-4, pp. 224-409, 2017.

[10] W. Cummins, "The impulse response function and ship motions," DTIC Document, Tech. Rep., 1962.

[11] J. Falnes, Ocean waves and oscillating systems: linear interactions including wave-energy extraction. Cambridge university press, 2002.

[12] K. Zhou and J. C. Doyle, Essentials of robust control. Prentice hall Upper Saddle River, NJ, 1998, vol. 104.

[13] C. A. Floudas and V. Visweswaran, "Quadratic optimization," in Handbook of global optimization. Springer, 1995, pp. 217-269.

[14] J. Wolfram, "On alternative approaches to linearization and Morison's equation for wave forces," in Proceedings of the Royal Society of London A: Mathematical, Physical and Engineering Sciences, vol. 455, no. 1988. The Royal Society, 1999, pp. 2957-2974.

[15] K. Hasselmann, "Measurements of wind wave growth and swell decay during the Joint North Sea Wave Project (JONSWAP)," Dtsch. Hydrogr. Z., vol. 8, p. 95, 1973. 